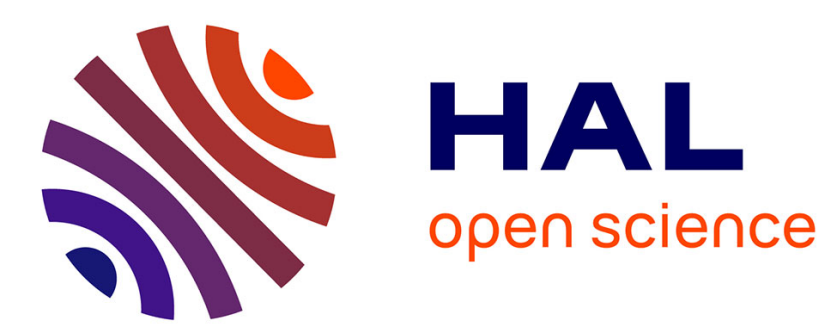

\title{
Reconstruction of nonstationary sound fields based on time domain plane wave superposition method
}

Xiao-Zheng Zhang, Jean-Hugh Thomas, Chuan-Xing Bi, Jean-Claude Pascal

\section{To cite this version:}

Xiao-Zheng Zhang, Jean-Hugh Thomas, Chuan-Xing Bi, Jean-Claude Pascal. Reconstruction of nonstationary sound fields based on time domain plane wave superposition method. Acoustics 2012, Apr 2012, Nantes, France. hal-00810673

\section{HAL Id: hal-00810673 https://hal.science/hal-00810673}

Submitted on 23 Apr 2012

HAL is a multi-disciplinary open access archive for the deposit and dissemination of scientific research documents, whether they are published or not. The documents may come from teaching and research institutions in France or abroad, or from public or private research centers.
L'archive ouverte pluridisciplinaire HAL, est destinée au dépôt et à la diffusion de documents scientifiques de niveau recherche, publiés ou non, émanant des établissements d'enseignement et de recherche français ou étrangers, des laboratoires publics ou privés. 


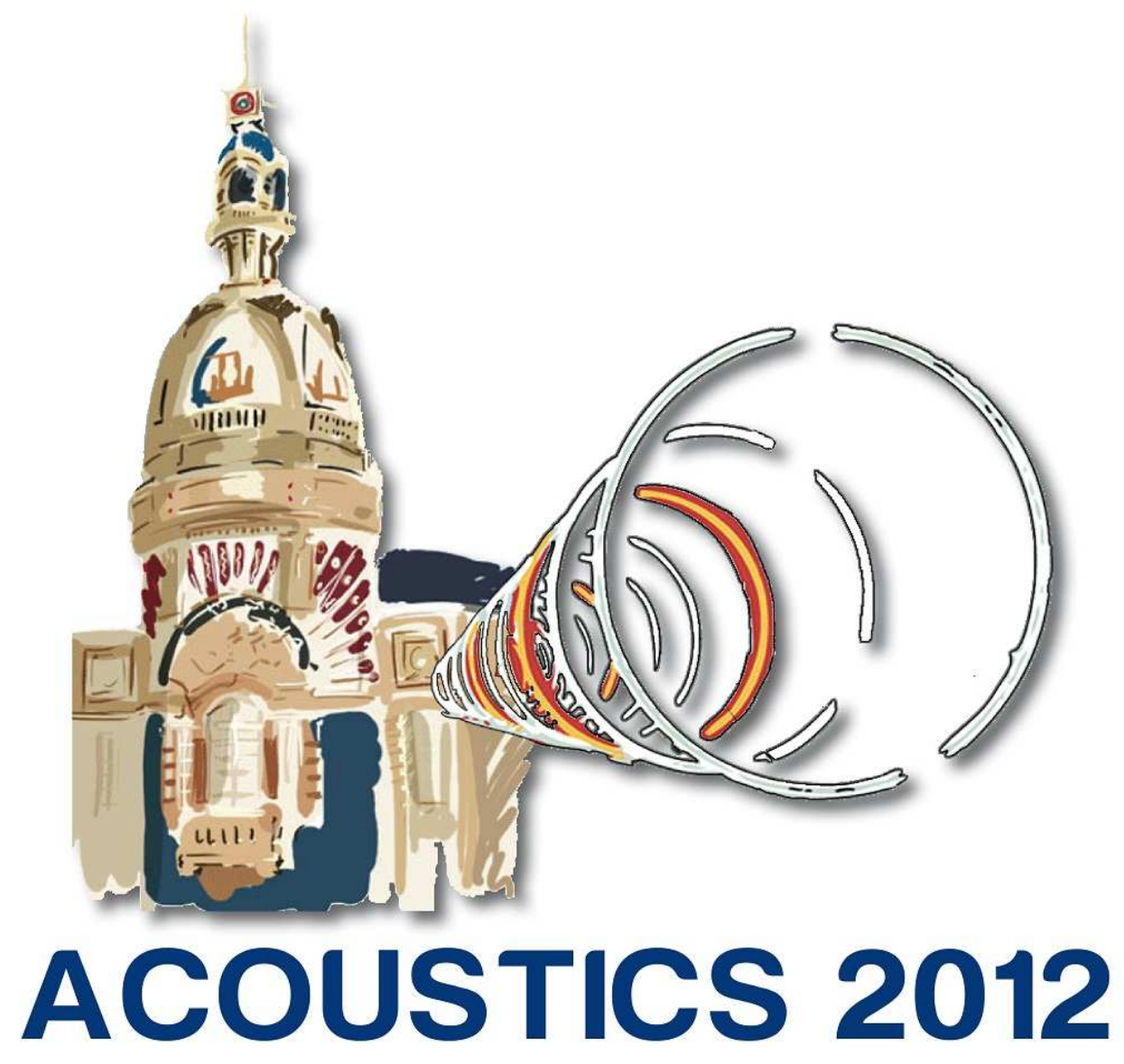

Reconstruction of nonstationary sound fields based on time domain plane wave superposition method

X.-Z. Zhang ${ }^{\mathrm{a}}$, J.-H. Thomas ${ }^{\mathrm{b}}$, C.-X. Bi ${ }^{\mathrm{a}}$ and J.-C. Pascal ${ }^{\mathrm{b}}$

${ }^{a}$ Institute of Sound and Vibration Research, Hefei University of Technology, 230009 Hefei, China

bLaboratoire d'acoustique de l'université du Maine, Bât. IAM - UFR Sciences Avenue Olivier Messiaen 72085 Le Mans Cedex 9

xzhengzhang@hotmail.com 
A time-domain plane wave superposition method (TD-PWSM) is proposed to reconstruct nonstationary sound fields. At each time step of this method, the time-wavenumber spectrum of the sound pressure on the virtual source plane is firstly estimated by using the measured sound pressure and the right pseudo-inverse of the timedomain propagation kernel matrix, and then the reconstruction is performed through a superposition of all the time convolutions between the estimated time-wavenumber spectrum of the sound pressure on the virtual source plane and the time-domain propagation kernel at each wavenumber. Since the inverse process at each time step is ill-conditioned, the Tikhonov regularization is introduced to obtain an appropriate solution. The method proposed provides the ability of continuously reconstructing time-dependent pressure signals and overcomes some errors due to the use of the two-dimensional spatial Fourier transforms, which is avoided. Numerical simulations demonstrate that it is feasible to reconstruct the nonstationary sound fields via TD-PWSM.

\section{Introduction}

Nearfield acoustic holography (NAH) $[1,2]$ is a wellknown technique for reconstructing three-dimensional sound fields based on discrete spatial Fourier transforms of sound pressure data measured over a finite area. However, it is usually applied to stationary sound fields. When the signals emitted by sources are nonstationary, NAH is unsuitable because the spatial sound fields have statistical properties which fluctuate with time. In order to reconstruct nonstationary sound fields, Time Domain Holography (TDH) [3] and Real-time Nearfield Acoustic Holography (RT-NAH) [4,5] are proposed. Nevertheless, either in TDH or in RT-NAH, the two-dimensional spatial Fourier transforms of the measured sound pressure are employed. In this paper a time domain plane wave superposition method (TD-PWSM) is proposed, which performs the reconstruction directly through a superposition of all the time convolutions between the estimated time-wavenumber spectrum of the sound pressure on the virtual source plane and the time-domain propagation kernel at each wavenumber, and needs no spatial Fourier transforms of the measured sound pressure, thus naturally avoids the errors and limitations (the window effects, etc.) associated with the spatial Fourier transforms. Similarly to RT-NAH, TDPWSM performs the reconstruction directly in the time domain, which also provides the advantage of continuously reconstructing time-dependent pressure signals. To examine the feasibility of reconstructing nonstationary sound fields by using TD-PWSM, a simulation is also presented in this paper.

\section{Theory of TD-PWSM}

\subsection{The formulas of TD-PWSM}

Assume that all sources are behind the calculation plane $z=z_{c}$. There exist a virtual source plane $z=z_{v}$ in the half space $z \leq z_{c}$ and a measurement plane $z=z_{m}$ with $N$ measurement points in the half space $z>z_{c}$, as shown in Fig. 1.

According to the forward propagation formulation of the sound pressure in the time-wavenumber domain as shown in Ref. 6, the time-wavenumber spectrum of the pressure on the measurement plane can be expressed as the convolution of that on the virtual source plane and the impulse response function, that is

$$
P\left(k_{x}, k_{y}, z_{m}, t\right)=P\left(k_{x}, k_{y}, z_{v}, t\right) * h\left(k_{x}, k_{y}, \Delta z_{m v}, t\right),
$$

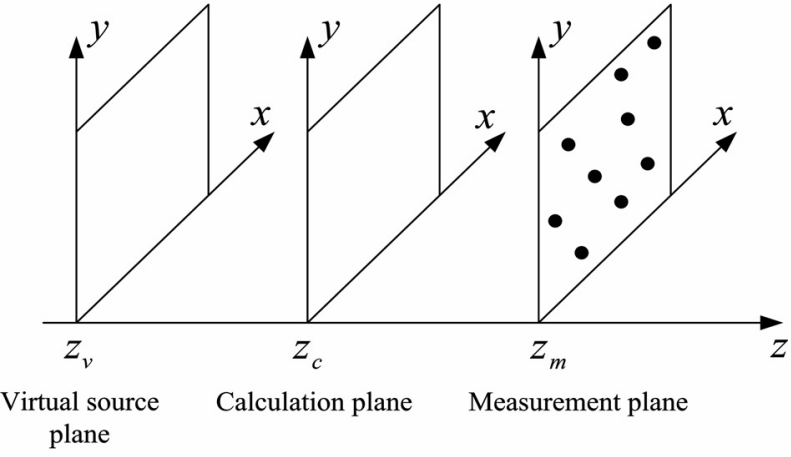

Figure 1: Geometry of the virtual source plane, the calculation plane and the measurement plane.

where the propagation distance $\Delta z_{m v}=z_{m}-z_{v}, h$ is the impulse response function and given by

$$
h\left(\Omega_{r}, \tau, t\right)=\delta(t-\tau)-\tau \Omega_{r} \frac{J_{1}\left(\Omega_{r} \sqrt{t^{2}-\tau^{2}}\right)}{\sqrt{t^{2}-\tau^{2}}} H(t-\tau) .
$$

In Eq. (2), the transition pulsation $\Omega_{r}=c \sqrt{k_{x}^{2}+k_{y}^{2}}$, the propagation delay $\tau=\Delta z_{m v} / c, c$ denotes the sound velocity, $\delta(t)$ denotes the Dirac delta function, $J_{1}$ denotes the Bessel function of the first kind and order 1 , and $H(t)$ denotes the Heaviside function.

Through setting $\mathbf{r}=(x, y)$ which represents a coordinate vector of any point in the space, $\mathbf{K}=\left(k_{x}, k_{y}\right)$ which represents a wavenumber vector, and applying the inverse two-dimensional spatial Fourier transforms with respect to $x$ and $y$ to Eq. (1), it yields

$$
\begin{aligned}
& p\left(\mathbf{r}, z_{m}, t\right)= \\
& \quad \frac{1}{(2 \pi)^{2}} \int_{-\infty}^{\infty} \int_{-\infty}^{\infty} P\left(\mathbf{K}, z_{v}, t\right) *\left[h\left(\mathbf{K}, \Delta z_{m v}, t\right) e^{-j(\mathbf{K} \cdot \mathbf{r})}\right] d \mathbf{K} .
\end{aligned}
$$

In Eq. (3), the double integral is discretized in the wavenumber domain, then $p\left(\mathbf{r}, z_{m}, t\right)$ is approximately given by

$$
\begin{aligned}
& p\left(\mathbf{r}, z_{m}, t\right) \approx \\
& \quad \sum_{l=1}^{L} P\left(\mathbf{K}_{l}, z_{v}, t\right) *\left[\frac{\Delta k_{x} \Delta k_{y}}{(2 \pi)^{2}} h\left(\mathbf{K}_{l}, \Delta z_{m v}, t\right) e^{-j\left(\mathbf{K}_{l} \bullet \mathbf{r}\right)}\right] .
\end{aligned}
$$


Here, $\left\{\mathbf{K}_{l}, l=1, \ldots L\right\}$ is the set of the sampling points in the wavenumber domain. $\Delta k_{x}$ and $\Delta k_{y}$ are the sampling spacing respectively for $k_{x}$ and $k_{y}$ wavenumbers. When $\Delta k_{x} \rightarrow 0, \Delta k_{y} \rightarrow 0$, and $L \rightarrow \infty$, the accurate value of $p\left(\mathbf{r}, z_{m}, t\right)$ can be obtained from Eq. (4). However, due to discrete computing, these conditions are never fulfilled. Meanwhile, since the evanescent waves corresponding to the larger wavenumbers rapidly decay with an increasing distance and the larger the wavenumber, the faster the decay, the tiny contributions associated with the larger wavenumbers on the sound pressure $p\left(\mathbf{r}, z_{m}, t\right)$ can be neglected. Thus the almost accurate value of $p\left(\mathbf{r}, z_{m}, t\right)$ can be obtained by giving $L$, a limited number of wavenumbers sampled once one ensures the wavenumber domain sampling area to cover all propagating waves and those evanescent waves which have significant amplitude in the measurement region.[7]

Defining the time-domain propagation kernel between the sound pressure on the measurement plane $p\left(\mathbf{r}, z_{m}, t\right)$ and the time-wavenumber spectrum of the sound pressure on the virtual source plane $P\left(\mathbf{K}_{l}, z_{v}, t\right)$ as

$$
\psi\left(\mathbf{K}_{l}, \mathbf{r}, \Delta z_{m v}, t\right)=\frac{\Delta k_{x} \Delta k_{y}}{(2 \pi)^{2}} h\left(\mathbf{K}_{l}, \Delta z_{m v}, t\right) e^{-j\left(\mathbf{K}_{l} \cdot \mathbf{r}\right)}
$$

Eq. (4) can be rewritten as

$$
p\left(\mathbf{r}, z_{m}, t\right)=\sum_{l=1}^{L} P\left(\mathbf{K}_{l}, z_{v}, t\right) * \psi\left(\mathbf{K}_{l}, \mathbf{r}, \Delta z_{m v}, t\right) .
$$

Similarly the time-dependent spatial sound field on the calculation plane $z=z_{c}$ is

$$
p\left(\mathbf{r}, z_{c}, t\right)=\sum_{l=1}^{L} P\left(\mathbf{K}_{l}, z_{v}, t\right) * \psi\left(\mathbf{K}_{l}, \mathbf{r}, \Delta z_{c v}, t\right)
$$

where $\Delta z_{c v}=z_{c}-z_{v}$.

Eqs. (6) and (7) constitute the basic formulas of the TDPWSM, which describe that the time-dependent sound pressure radiated by the actual sources as a superposition of all the time convolutions between the time-wavenumber spectrum of the sound pressure on the virtual source plane and the time-domain propagation kernel at each wavenumber. If the time-wavenumber spectrum $P\left(\mathbf{K}_{l}, z_{v}, t\right)$ in Eq. (6) can be estimated properly by fitting the measured sound pressure $p\left(\mathbf{r}, z_{m}, t\right)$, and then is substituted to Eq. (7), the time-dependent spatial sound field on the calculation plane $p\left(\mathbf{r}, z_{c}, t\right)$ would be directly obtained. Compared with those in TDH and RT-NAH, this reconstruction process does not use the two-dimensional spatial Fourier transforms of the measured sound pressure.

\subsection{The reconstruction process}

In order to implement Eqs. (6) and (7), the discrete time variable $t_{i}$ such as

$$
t_{i}=(i-1) \Delta t, \quad i=1,2, \cdots I,
$$

is used where $\Delta t$ is the sampling period. According to Eqs. (2) and (5), the time-domain propagation kernel $\psi\left(\mathbf{K}_{l}, \mathbf{r}, \Delta z_{m v}, t\right)$ is equal to zero for $t<\tau$ caused by the fact that the impulse response function $h$ equals to zero for $t<\tau$. Consider that $t_{k}$ is the nearest time step more than or equal to $\tau$. According to the discrete convolution formula, at time step $t_{k}$ the spatial sound pressure on the measurement plane $z=z_{m}$ is

$$
p\left(\mathbf{r}, z_{m}, t_{k}\right)=\sum_{l=1}^{L} P\left(\mathbf{K}_{l}, z_{v}, t_{1}\right) \times \psi\left(\mathbf{K}_{l}, \mathbf{r}, \Delta z_{m v}, t_{k}\right) \times \Delta t .
$$

Here for the sake of simplicity, $\Delta t$ is omitted in the following formulas. The matrix formulation

$$
\mathbf{p}_{m}^{k}=\boldsymbol{\Psi}_{m}^{k} \mathbf{P}^{1}
$$

where

$$
\begin{aligned}
& \mathbf{p}_{m}^{k}=\left[p\left(\mathbf{r}_{1}, z_{m}, t_{k}\right) \cdots \quad \cdots\left(\mathbf{r}_{N}, z_{m}, t_{k}\right)\right]^{T}, \\
& \mathbf{P}^{1}=\left[P\left(\mathbf{K}_{1}, z_{v}, t_{1}\right) \quad \cdots \quad P\left(\mathbf{K}_{L}, z_{v}, t_{1}\right)\right]^{T}, \\
& \boldsymbol{\Psi}_{m}^{k}=\left[\begin{array}{ccc}
\psi\left(\mathbf{K}_{1}, \mathbf{r}_{1}, \Delta z_{m v}, t_{k}\right) & \cdots & \psi\left(\mathbf{K}_{L}, \mathbf{r}_{1}, \Delta z_{m v}, t_{k}\right) \\
\vdots & \vdots & \vdots \\
\psi\left(\mathbf{K}_{1}, \mathbf{r}_{N}, \Delta z_{m v}, t_{k}\right) & \cdots & \psi\left(\mathbf{K}_{L}, \mathbf{r}_{N}, \Delta z_{m v}, t_{k}\right)
\end{array}\right],
\end{aligned}
$$

has the advantage to describe Eq. (9) for $N$ measurement points of coordinate $\mathbf{r}_{i}(l=1, \ldots L)$. Note that $\boldsymbol{\Psi}_{m}^{k}$ is a $N \times L$ matrix. In practice, the number of measurement points $N$ is often smaller than the number of discrete wavenumbers $L$. The smallest 2-norm solution of Eq. (10) can be obtained using the right pseudo-inverse of matrix $\boldsymbol{\Psi}_{m}^{k}$, which is expressed as

$$
\widetilde{\mathbf{P}}^{1}=\left[\boldsymbol{\Psi}_{m}^{k}\right]^{+R} \mathbf{p}_{m}^{k},
$$

where $\left[\boldsymbol{\Psi}_{m}^{k}\right]^{+R}$ represents the right pseudo-inverse of matrix $\boldsymbol{\Psi}_{m}^{k}$ and is given by

$$
\left[\boldsymbol{\Psi}_{m}^{k}\right]^{+R}=\boldsymbol{\Psi}_{m}^{k}{ }_{m}^{H}\left(\boldsymbol{\Psi}_{m}^{k} \boldsymbol{\Psi}_{m}^{k}{ }^{H}\right)^{-1}
$$

Once the smallest 2-norm solution $\widetilde{\mathbf{P}}^{1}$ is solved, the pressure at any point $\mathbf{r}^{\prime}$ on the calculation surface at the first time step $t_{1}$ can be deduced as

$$
p\left(\mathbf{r}^{\prime}, z_{c}, t_{1}\right)=\boldsymbol{\Psi}_{c}^{1} \widetilde{\mathbf{P}}^{1},
$$

where

$$
\boldsymbol{\Psi}_{c}^{1}=\left[\psi\left(\mathbf{K}_{1}, \mathbf{r}^{\prime}, \Delta z_{c v}, t_{1}\right) \quad \cdots \quad \psi\left(\mathbf{K}_{L}, \mathbf{r}^{\prime}, \Delta z_{c v}, t_{1}\right)\right] .
$$

And so forth, the solution at the $i$ th time step $t_{i}$ $(i=2,3, \cdots I-k+1)$ is given by 


$$
\widetilde{\mathbf{P}}^{i}=\left[\boldsymbol{\Psi}_{m}^{k}\right]^{+R}\left(\mathbf{p}_{m}^{i+k-1}-\boldsymbol{\Psi}_{m}^{k+1} \widetilde{\mathbf{P}}^{i-1}-\cdots-\boldsymbol{\Psi}_{m}^{i+k-1} \widetilde{\mathbf{P}}^{1}\right) .
$$

Thus the pressure is obtained at any point $\mathbf{r}^{\prime}$ on the calculation surface at the $i$ th time step $t_{i}$

$$
p\left(\mathbf{r}^{\prime}, z_{c}, t_{i}\right)=\Psi_{c}^{1} \widetilde{\mathbf{P}}^{i}+\Psi_{c}^{2} \widetilde{\mathbf{P}}^{i-1}+\cdots+\Psi_{c}^{i} \widetilde{\mathbf{P}}^{1}
$$

where

$$
\boldsymbol{\Psi}_{c}^{i}=\left[\psi\left(\mathbf{K}_{1}, \mathbf{r}^{\prime}, \Delta z_{c v}, t_{i}\right) \quad \cdots \quad \psi\left(\mathbf{K}_{L}, \mathbf{r}^{\prime}, \Delta z_{c v}, t_{i}\right)\right] .
$$

In Eqs. (11) and (15), the direct use of the right pseudoinverse of matrix $\boldsymbol{\Psi}_{m}^{k}$ leads to an inappropriate solution because the inversion is often ill-conditioned. In order to obtain an appropriate solution, the Tikhonov regularization [8] is implemented. Then the smallest 2-norm solution at the $i$ th time step $t_{i}(i=1,2, \cdots I-k+1)$ becomes

$$
\widetilde{\mathbf{P}}_{\lambda}^{i}=\left[\boldsymbol{\Psi}_{m}^{k}\right]_{\lambda}^{+R}\left(\mathbf{p}_{m}^{i+k-1}-\boldsymbol{\Psi}_{m}^{k+1} \widetilde{\mathbf{P}}^{i-1}-\cdots-\boldsymbol{\Psi}_{m}^{i+k-1} \widetilde{\mathbf{P}}^{1}\right),
$$

where $\widetilde{\mathbf{P}}_{\lambda}^{i}$ denotes the regularized solution, and

$$
\left[\boldsymbol{\Psi}_{m}^{k}\right]_{\lambda}^{+R}=\boldsymbol{\Psi}_{m}^{k^{H}}\left(\boldsymbol{\Psi}_{m}^{k} \boldsymbol{\Psi}_{m}^{k^{H}}+\lambda \mathbf{I}\right)^{-1}
$$

The regularization parameter $\lambda$ is estimated by the Generalized Crossed Validation (GCV).[9]

\section{Numerical simulations}

The setup of the numerical simulations is shown in Fig. 2. The sources are composed of two monopoles, locating at $M_{1}(0.18 \mathrm{~m}, 0.18 \mathrm{~m}, 0 \mathrm{~m})$ and $M_{2}(0.36 \mathrm{~m}, 0.36 \mathrm{~m}, 0 \mathrm{~m})$. $M_{1}$ generates a nonstationary signal with a linear frequency modulation in the $[200,1800] \mathrm{Hz}$ band and a Gaussian amplitude modulation. $M_{2}$ radiates another nonstationary signal - Morlet wavelet defined by

$$
s(t)=\cos \left(2 \pi f_{0} t\right) e^{-t^{2} / 2},
$$

with $f_{0}=800 \mathrm{~Hz}$. The measurement plane located at $z_{m}=0.13 \mathrm{~m}$ provides $7 \times 7$ measurement points, and the spacing of measurement points in both $x$ and $y$ directions is set to $a=0.09 \mathrm{~m}$. The calculation plane is located at $z_{c}=0.09 \mathrm{~m}$, and the virtual source plane is located at $z_{v}=0.06 \mathrm{~m}$. The emitted signals are sampled at a frequency $f_{e}=34400 \mathrm{~Hz}$ providing 256 samples. According to the Nyquist theorem, the wavenumber domain sampling area is given as $[-\pi / a, \pi / a]$. Basing on the comprehensive consideration of the calculation precision and the computational efficiency, the wavenumber domain sampling spacing is given as $\pi /(18 a)$. A Gaussian white noise with a signal-to-noise ratio (SNR) of $20 \mathrm{~dB}$ is also added to the simulated signals.

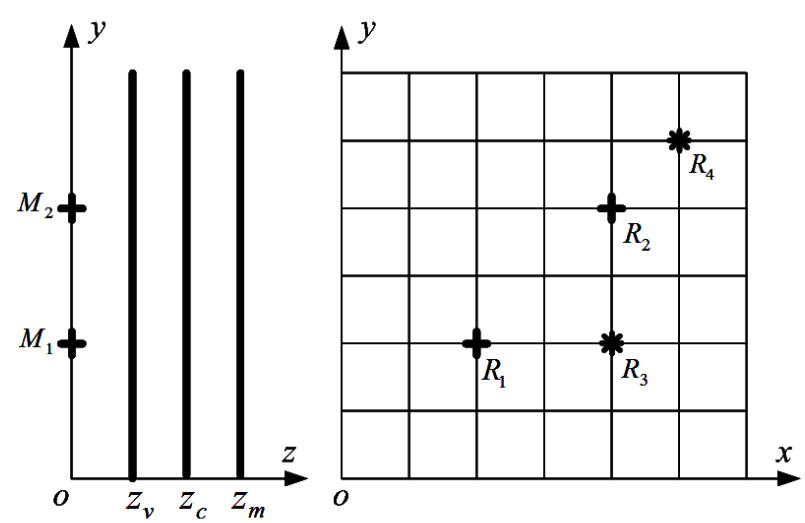

Figure 2: Geometric description of the virtual source plane, the calculation plane and the measurement plane. Points $R_{1}$ and $R_{2}$ marked with + , stand for the points facing the source $M_{1}$ and the source $M_{2}$ respectively. Points $R_{3}$ and $R_{4}$ marked with $*$, stand for the points not facing sources.

For the sake of assessing the relevance of the proposed method in the time domain, four space points on the calculation plane are chosen, and their positions are $R_{1}(0.18 \mathrm{~m}, 0.18 \mathrm{~m}, 0.09 \mathrm{~m}), R_{2}(0.36 \mathrm{~m}, 0.36 \mathrm{~m}, 0.09 \mathrm{~m})$, $R_{3}(0.36 \mathrm{~m}, 0.18 \mathrm{~m}, 0.09 \mathrm{~m}), R_{4}(0.45 \mathrm{~m}, 0.45 \mathrm{~m}, 0.09 \mathrm{~m})$. As shown in Fig. $2, R_{1}$ and $R_{2}$ are facing the source $M_{1}$ and the source $M_{2}$ respectively, while $R_{3}$ and $R_{4}$ are selected not facing the sources. Fig. 3 shows the comparisons between the calculated pressure and the theoretical pressure at these points in the time domain. It is demonstrated that the calculated results provide accurate phases and good magnitudes at all four points. (a) R1

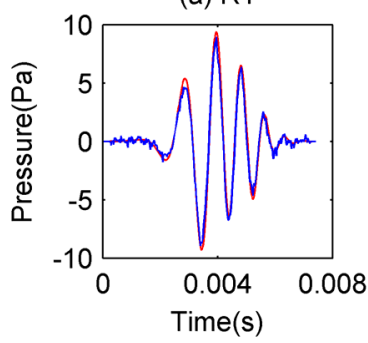

(c) R3

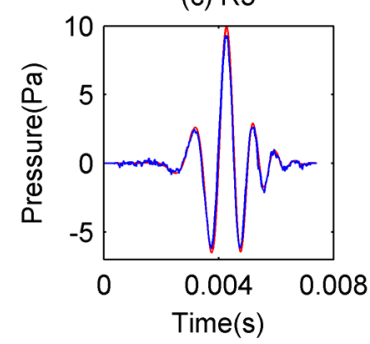

(b) R2

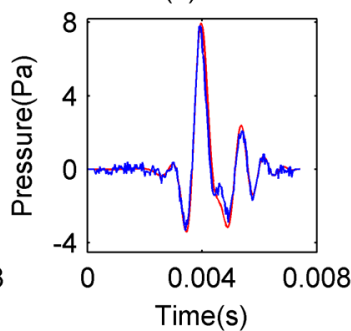

(d) R4

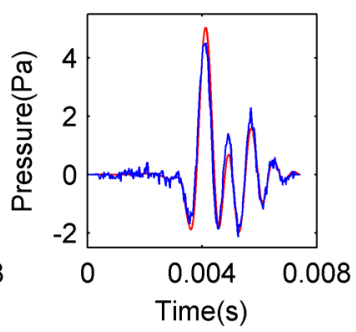

Figure 3: Comparison between the time-dependent pressure signals on the calculation plane in points $R_{1}(\mathrm{a}), R_{2}(\mathrm{~b})$, $R_{3}$ (c), and $R_{4}(\mathrm{~d})$ : the theoretical pressure (red line), and the calculated pressure (blue line). 
In order to comment more objectively the results calculated in the time domain, two time indicators $T_{1}$ and $T_{2}$ are computed for a point $\left(x_{i}, y_{j}\right)$ on the calculation plane. They are defined by

$$
T_{1}\left(x_{i}, y_{j}\right)=\frac{\left\langle p_{t}\left(x_{i}, y_{j}, z_{c}, t\right) p_{c}\left(x_{i}, y_{j}, z_{c}, t\right)\right\rangle_{t}}{\sqrt{\left.<p_{t}^{2}\left(x_{i}, y_{j}, z_{c}, t\right)>_{t}<p_{c}^{2}\left(x_{i}, y_{j}, z_{c}, t\right)\right\rangle_{t}}},
$$

$$
T_{2}\left(x_{i}, y_{j}\right)=\frac{\left|\sqrt{\left\langle p_{t}^{2}\left(x_{i}, y_{j}, z_{c}, t\right)\right\rangle_{t}}-\sqrt{\left\langle p_{c}^{2}\left(x_{i}, y_{j}, z_{c}, t\right)\right\rangle_{t}}\right|}{\sqrt{\left\langle p_{t}^{2}\left(x_{i}, y_{j}, z_{c}, t\right)\right\rangle_{t}}}
$$

$<>_{t}$ denotes the time averaged value. $p_{t}\left(x_{i}, y_{j}, z_{c}, t\right)$ is the theoretical time-dependent pressure and $p_{c}\left(x_{i}, y_{j}, z_{c}, t\right)$ is the calculated time-dependent pressure. $T_{1}$ and $T_{2}$ are sensitive to the phase differences and the magnitude differences between $p_{t}\left(x_{i}, y_{j}, z_{c}, t\right)$ and $p_{c}\left(x_{i}, y_{j}, z_{c}, t\right)$, respectively. Phase accuracy gives $T_{1}$ in the neighbourhood of 1 , and magnitude accuracy gives $T_{2}$ near 0 . The values of both indicators $T_{1}$ and $T_{2}$ are computed for each point on the calculation plane. The map of indicator $T_{1}$ with the 0.98 contour line is shown in Fig. 4(a) and the map of indicator $T_{2}$ with the 0.1 contour line is shown in Fig. 4(b). In Fig. 4(a), the values of indicator $T_{1}$ at most points are greater than 0.98 , which indicates that the phase of the calculated pressure matches well with that of the theoretical pressure. Fig. 4(b) shows that the values of indicator $T_{2}$ below 0.1 mainly appear the area far away from $R_{1}$ and $R_{2}$, where provides the better magnitude accuracy. The values of indicator $T_{1}$ at marked points $R_{1}(+), R_{2}(+), R_{3}(*)$, and $R_{4}(*)$ are $0.992,0.982,0.993$, and 0.974 , respectively. The values of indicator $T_{2}$ at marked points $R_{1}(+), R_{2}(+)$, $R_{3}(*)$, and $R_{4}(*)$ are $0.081,0.105,0.057$, and 0.046 , respectively. (a) Time indicator T1

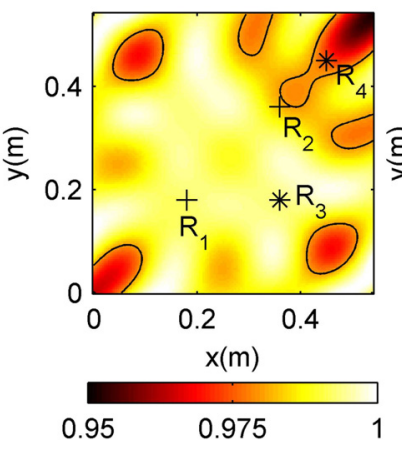

(b) Time indicator T2

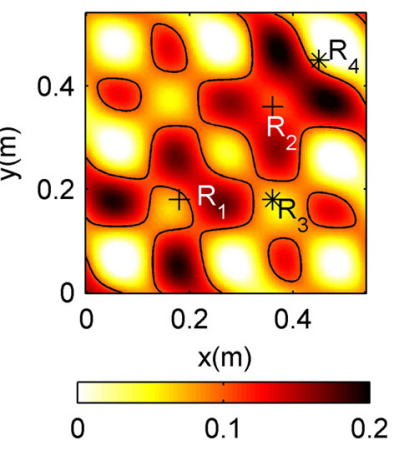

Figure 4: Spatial maps for indicator $T_{1}$ (a) with a contour line at the value 0.98 and for indicator $T_{2}$ (b) with a contour line at the value 0.1 . The points $R_{1}(+), R_{2}(+), R_{3}(*)$, and $R_{4}(*)$ are marked.
Similarly, to highlight the relevance of the proposed method in the space domain, two time instants $\left(t_{1}=3.9 \mathrm{~ms}\right.$ and $t_{2}=5.6 \mathrm{~ms}$ ) are selected. Figures 5(a) and 5(c) show the theoretical spatial pressure field and the calculated spatial pressure field at $t_{1}=3.9 \mathrm{~ms}$, respectively, where the acoustic field is dominated by the acoustic signals radiated by sources $M_{1}$ and $M_{2}$. Figures 5(b) and 5(d) show the same spatial pressure fields but at $t_{2}=5.6 \mathrm{~ms}$, where the source $M_{1}$ radiates with a high level and $M_{2}$ with a low level. From the comparison of the theoretical spatial pressure field and the calculated spatial pressure field, it is demonstrated that the proposed method provides a means of visualizing the spatial pressure field when the sound field fluctuates with time. (a) $p_{t}$ at $3.9 \mathrm{~ms}$

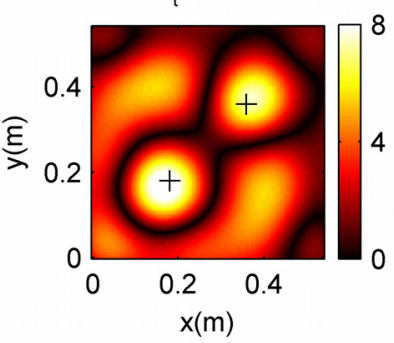

(c) $p_{c}$ at $3.9 \mathrm{~ms}$

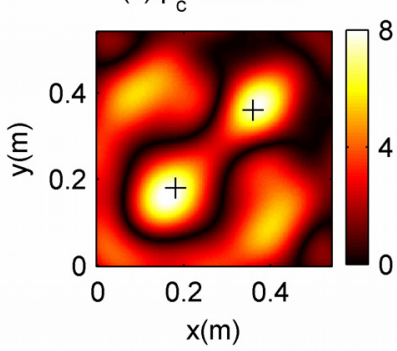

(b) $p_{t}$ at $5.6 \mathrm{~ms}$

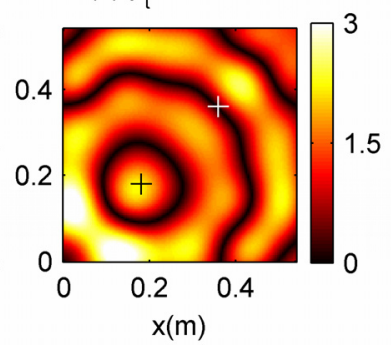

(d) $p_{c}$ at $5.6 \mathrm{~ms}$

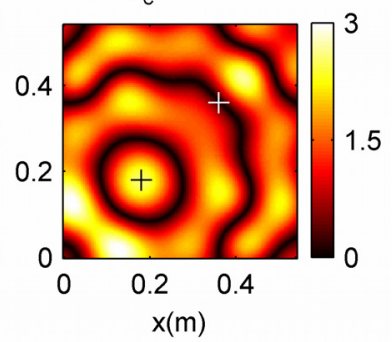

Figure 5: Theoretical spatial pressure field at $t_{1}=3.9 \mathrm{~ms}$ (a) and at $t_{2}=5.6 \mathrm{~ms}(\mathrm{~b})$, versus, calculated spatial pressure field at $t_{1}=3.9 \mathrm{~ms}(\mathrm{c})$ and at $t_{2}=5.6 \mathrm{~ms}(\mathrm{~d})$. The lower left marked location is facing the source $M_{1}$ and the upper right marked location is facing the source $M_{2}$.

In order to evaluate the quality of the results calculated in the space domain, a relative spatial error criterion is introduced, defined by

$$
E_{x, y}^{r}\left(t_{i}\right)=\frac{\sqrt{\left.<\left(p_{c}\left(x, y, t_{i}\right)-p_{t}\left(x, y, t_{i}\right)\right)^{2}\right\rangle_{s}}}{\sqrt{\left.<p_{t}^{2}\left(x, y, t_{i}\right)\right\rangle_{s}}},
$$

which corresponds to the root mean square error between the calculated pressure $p_{c}\left(x, y, t_{i}\right)$ and the theoretical pressure $p_{t}\left(x, y, t_{i}\right)$ at a given time $t_{i}$, and \langle\rangle$_{s}$ denotes the spatial averaged value. The time evolution of the root mean square error is shown in Fig. 6, from which it can be seen that at most time instants the values of the root mean square error are smaller than 0.5 . High values of the error are obtained at the edges of the signal due to the fact that the theoretical pressure field supplies the denominator of Eq. (23) with very low values at these time instants. The 
values of the spatial error calculated at $t_{1}=3.9 \mathrm{~ms}$ and at $t_{2}=5.6 \mathrm{~ms}$ are $0.194,0.201$, respectively.

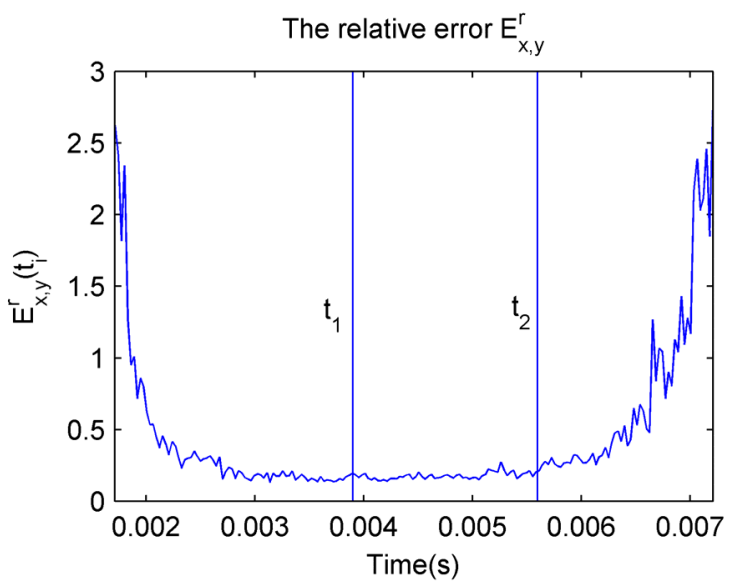

Figure 6: Time evolution of the spatial error criterion. The vertical lines indicate the time instants chosen at $t_{1}=3.9 \mathrm{~ms}$ and at $t_{2}=5.6 \mathrm{~ms}$.

\section{Conclusion}

To reconstruct the nonstationary sound fields fluctuating with time, a time domain plane wave superposition method was proposed, which realizes the reconstruction through a superposition of all the time convolutions between the estimated time-wavenumber spectrum of the sound pressure on the virtual source plane and the time-domain propagation kernel at each wavenumber. Numerical simulations have demonstrated the ability of the method to calculate the time-independent pressure at each space point and visualize the spatial pressure field at each time instant. In theory, compared with TDH and RT-NAH, the method proposed has two advantages due to the fact it does not require to perform the two-dimensional spatial Fourier transforms of the measured sound pressure. One is to avoid the errors associated with the spatial Fourier transforms. The other is to remove the limit of regular microphone array, that is to say the microphone array employed by TDPWSM could be irregular, such as cross array, circular array, random array, etc. These two advantages will be discussed using examples in the future.

\section{Acknowledgments}

This work was supported by National Natural Science Foundation of China (Grant Nos. 10974040 and 51105126), the Program for New Century Excellent Talents in University (Grant No. NCET-08-0767), and the Research Fund for the Doctoral Program of Higher Education (Grand No. 20100111110007).

\section{References}

[1] J. D. Maynard, E. G. Williams, Y. Lee, "Nearfield acoustic holography I: Theory of generalized holography and development of NAH", J. Acoust. Soc. Am. 78(4), 1395-1413 (1985)

[2] J. D. Maynard, E. G. Williams, "Nearfield acoustic holography (NAH) II: Holographic reconstruction algorithms and computer implementation", J. Acoust. Soc. Am. 81(5), 1307-1322 (1987)

[3] J. Hald, "Time domain acoustical holography and its applications," Sound and Vibration 35, 16-24 (2001)

[4] J.-H. Thomas, V. Grulier, S. Paillasseur, J.-C. Pascal, J.-C. Le Roux, "Real-time near-field acoustic holography for continuously visualizing nonstationary acoustic fields", J. Acoust. Soc. Am. 128(6), 3554-3567 (2010)

[5] S. Paillasseur, J.-H. Thomas, J.-C. Pascal, "Regularization for improving the deconvolution in real-time near-field acoustic holography", J. Acoust. Soc. Am. 129(6), 3777-3787 (2011)

[6] V. Grulier, S. Paillasseur, J.-H. Thomas, J.-C. Pascal, J.-C. Le Roux, "Forward propagation of time evolving acoustic pressure : Formulation and investigation of the impulse response in time-wavenumber domain", $J$. Acoust. Soc. Am. 126(5), 2367-2378 (2009)

[7] J. Hald, "Basic theory and properties of statistically optimized near-field acoustical holography", J. Acoust. Soc. Am. 125(4), 2105-2120 (2009)

[8] A. Tikhonov, "Solutions of incorrectly formulated problems and the regularization method", Soviet. Math. Dokl. 4, 1035-1038 (1963)

[9] P. C. Hansen, Rank-deficient and discrete ill-posed problems, SIAM, Philadelphia (1998) 